Tropical Journal of Pharmaceutical Research August 2012; 11 (4): 611-620

(C) Pharmacotherapy Group, Faculty of Pharmacy, University of Benin Benin City, 300001 Nigeria.

All rights reserved.

\title{
Synthesis and Antitubercular Activity of Some Novel Thiazolidinone Derivatives
}

\author{
Himaja Malipeddi ${ }^{1}$, Asif A Karigar ${ }^{2 *}$, Venkata Ramana Malipeddi ${ }^{2}$ and \\ Mukesh S Sikarwar ${ }^{3}$ \\ ${ }^{1}$ Pharmaceutical Chemistry Division, School of Advanced Sciences, VIT University, Vellore 632 014, ${ }^{2}$ Maratha \\ Mandal's College of Pharmacy, Belgaum 590016, Karnataka, ${ }^{3}$ KLEU College of Pharmacy, Belgaum, Karnataka, \\ India.
}

\begin{abstract}
Purpose: To synthesize and characterize novel thiazolidinone derivatives and screen them for antitubercular activity.

Methods: A series of twelve novel thiazolidinones 4a-I have been synthesized by cyclocondensation of various Schiff bases of amino thiadiazole with thioglycollic acid. Various Schiff bases 3a-I were synthesized by condensation of 2-amino-5-aryl-5H-thiazolo[4,3-b]-l,3,4-thiadiazole with various aryl aldehydes. The synthesized compounds were characterized by FTIR, ${ }^{1} \mathrm{H}-\mathrm{NMR},{ }^{13} \mathrm{C}-\mathrm{NMR}$ and mass spectrometry. Docking studies were carried out for the synthesized compounds which were also evaluated for in vitro anti-tubercular activity at a concentration of $0.1-100.0 \mu \mathrm{g} / \mathrm{mL}$ by Microplate Blue Alamar Assay method. Pyrazinamide and streptomycin were used as standard antitubercular agents.

Results: The synthesized compounds showed good docking score, compared to standard drugs. Two of the compounds (labelled $\mathbf{4 f}$ and 4i) showed higher antitubercular activity than the standards (pyrazinamide and streptomycin) while compounds four others compounds (labeled $\mathbf{4 b}, \mathbf{4 c}, \mathbf{4 e}, \mathbf{4 h}, \mathbf{4 k}$ and 4I) showed comparable activity to pyrazinamide but greater activity than streptomycin.

Conclusion: We report the successful synthesis of novel thiazolidinones, as well as their spectral characterization, docking properties and in vitro antitubercular activities which, for some, are superior to currently used anti-tubercular agents.
\end{abstract}

Keywords: Thiadiazole, Schiff base, Thiazolidinone, Anti-tubercular activity, Docking 


\section{INTRODUCTION}

Despite the ready availability of effective treatments, tuberculosis remains a major public health threat worldwide. The emergence of drug-resistant strains of Mycobacterium tuberculosis, particularly multiple drug resistant strains [1-4], has complicated treatment protocols and raises the concern that tuberculosis may once again become an incurable disease. For this reason, it is critical to discover new drugs acting with a mechanism different from those presently in use.

Small ring heterocycles containing nitrogen, sulfur and oxygen have been under investigation for a long time because of their important medicinal properties. Among this type of molecules, 1,3,4-thiadiazoles and 4thiazolidinones were shown to have various important biological activities such as antibacterial, antifungal, antiviral, diuretic, tuberculostatic, anti-HIV, antihistaminic, anticancer, anticonvulsant, anti-inflammatory and analgesic properties [5-12].

In order to further assess the pharmacological profile of this class of compounds, it was thought worthwhile to synthesize some new congeners of heterocycles by incorporating thiadiazole and thiazolidinone moieties in a single molecular framework. The present work deals with the synthesis of these compounds as well as their anti-tubercular screening.

\section{EXPERIMENTAL}

\section{Materials}

Analytical grade solvents and commercially available reagents were used without further purification. All chemicals were obtained from Spectrochem Ltd (Mumbai, India). Column chromatography was carried out over silica gel (60 - 120 mesh), purchased from Sisco Research Laboratories Pvt Ltd. Mumbai, India. Melting point was determined in a programmable melting point apparatus
(Servewell Instruments Pvt Ltd). Fourier transform infrared spectroscopy (FTIR) in $\mathrm{KBr}$ disk were recorded from 4000 to $400 \mathrm{~cm}^{-1}$ on a Shimadzu FT-IR spectrometer (model no. 00518). ${ }^{1} \mathrm{H}$ and ${ }^{13} \mathrm{C}$ nuclear magnetic resonance (NMR) spectra were recorded on AMX $400-\mathrm{MHz}$ and $500-\mathrm{MHz}$ Bruker spectrometer in DMSO- $d_{6}$ or $\mathrm{CDCl}_{3}$ using tetramethylsilane (TMS) as an internal standard. Chemical shifts are given in $\delta$ relative to TMS while the coupling constants are given in $\mathrm{Hz}$. Mass spectra were recorded using Agilent 1100 MSD spectrometer in electrospray mode.

\section{Synthesis}

All the compounds were synthesized according to the scheme shown in Figure 1. The purity of the compounds was determined on TLC plates using silica gel $G$ as a stationary phase and iodine vapor as visualizing agent.

\section{Preparation of 2-amino-5-aryl-5H- thiazolo[4,3-b]-I,3,4-thiadiazole (1)}

2-amino-5-aryl-5H-thiazolo[4,3-b]-I,3,4thiadiazoles were prepared according to the procedure reported in the literature [13]. An aromatic aldehyde $(0.02 \mathrm{M})$ and thioglycolic acid (0.02M) were mixed, and after 10-15 $\mathrm{min}$. Thiosemicarbazide $(0.022 \mathrm{M})$ was added; then $10 \mathrm{~mL}$ of concentrated $\mathrm{H}_{2} \mathrm{SO}_{4}$ was added in portions upon cooling. The mixture was homogenized and left for $18-24 \mathrm{~h}$ at -20 ${ }^{\circ} \mathrm{C}$. The reaction mass was treated with $30-$ $50 \mathrm{~g}$ ice, the precipitated solid decanted, water added, and the resulting suspension neutralized with $40 \% \mathrm{NaOH}$ solution. The synthesized compound was recrystallized from aqueous dioxane solution.

\section{General procedure for the synthesis of compounds 3a-3l}

To a stirred solution of compound $1(0.01 \mathrm{M})$ in ethanol $(50 \mathrm{ml})$ containing sulphuric acid (2 $\mathrm{ml}$ ) was added appropriate aromatic aldehyde $(0.01 \mathrm{M})$ and the mixture refluxed for 

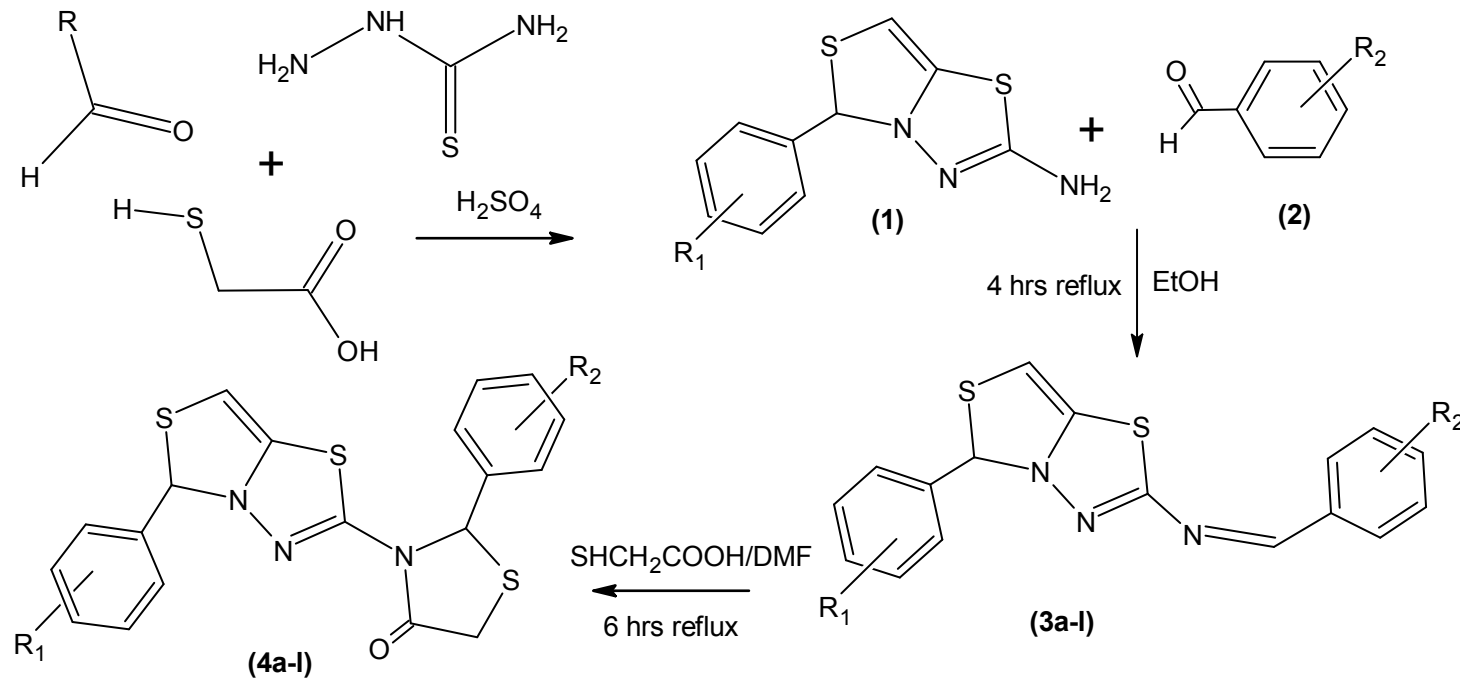

$$
\begin{aligned}
& \mathrm{R}_{1}=-\mathrm{H}, \mathrm{R}_{2}=-\mathrm{H},(4 \mathrm{a}) \\
& \mathrm{R}_{1}=-\mathrm{H}, \mathrm{R}_{2}=2-\mathrm{Cl},(4 \mathrm{~b}) \\
& \mathrm{R}_{1}=-\mathrm{H}, \mathrm{R}_{2}=4-\mathrm{Cl},(4 \mathrm{c}) \\
& \mathrm{R}_{1}=4-\mathrm{CH}_{3}, \mathrm{R}_{2}=\mathrm{H},(4 \mathrm{~d}) \\
& \mathrm{R}_{1}=4-\mathrm{CH}_{3}, \mathrm{R}_{2}=2-\mathrm{CH}_{3},(4 \mathrm{e}) \\
& \mathrm{R}_{1}=4-\mathrm{CH}_{3}, \mathrm{R}_{2}=4-\mathrm{CH}_{3},(4 \mathrm{f}) \\
& \mathrm{R}_{1}=4-\mathrm{OH}, \mathrm{R}_{2}=-\mathrm{H},(4 \mathrm{~g}) \\
& \mathrm{R}_{1}=4-\mathrm{OH}, \mathrm{R}_{2}=2-\mathrm{OH},(4 \mathrm{~h}) \\
& \mathrm{R}_{1}=4-\mathrm{OH}, \mathrm{R}_{2}=4-\mathrm{OH},(4 \mathrm{i}) \\
& \mathrm{R}_{1}=4-\mathrm{N}\left(\mathrm{CH}_{3}\right)_{2}, \mathrm{R}_{2}=\mathrm{H},(4 \mathrm{j}) \\
& \mathrm{R}_{1}=4-\mathrm{N}\left(\mathrm{CH}_{3}\right)_{2}, \mathrm{R}_{2}=2-\mathrm{OCH}_{3},(4 \mathrm{k}) \\
& \mathrm{R}_{1}=4-\mathrm{N}\left(\mathrm{CH}_{3}\right)_{2}, \mathrm{R}_{2}=4-\mathrm{OCH}_{3},(4 \mathrm{I})
\end{aligned}
$$

$$
\begin{aligned}
& \mathrm{R}_{1}=-\mathrm{H}, \mathrm{R}_{2}=-\mathrm{H},(3 \mathrm{a}) \\
& \mathrm{R}_{1}=-\mathrm{H}, \mathrm{R}_{2}=2-\mathrm{Cl},(3 \mathrm{~b}) \\
& \mathrm{R}_{1}=-\mathrm{H}, \mathrm{R}_{2}=4-\mathrm{Cl},(3 \mathrm{c}) \\
& \mathrm{R}_{1}=4-\mathrm{CH}_{3}, \mathrm{R}_{2}=\mathrm{H},(3 \mathrm{~d}) \\
& \mathrm{R}_{1}=4-\mathrm{CH}_{3}, \mathrm{R}_{2}=2-\mathrm{CH}_{3},(3 \mathrm{e}) \\
& \mathrm{R}_{1}=4-\mathrm{CH}_{3}, \mathrm{R}_{2}=4-\mathrm{CH}_{3},(3 \mathrm{f}) \\
& \mathrm{R}_{1}=4-\mathrm{OH}, \mathrm{R}_{2}=-\mathrm{H},(3 \mathrm{~g}) \\
& \mathrm{R}_{1}=4-\mathrm{OH}, \mathrm{R}_{2}=2-\mathrm{OH},(3 \mathrm{~h}) \\
& \mathrm{R}_{1}=4-\mathrm{OH}, \mathrm{R}_{2}=4-\mathrm{OH},(3 \mathrm{i}) \\
& \mathrm{R}_{1}=4-\mathrm{N}\left(\mathrm{CH}_{3}\right)_{2}, \mathrm{R}_{2}=\mathrm{H},(3 \mathrm{j}) \\
& \mathrm{R}_{1}=4-\mathrm{N}\left(\mathrm{CH}_{3}\right)_{2}, \mathrm{R}_{2}=2-\mathrm{OCH}_{3},(3 \mathrm{k}) \\
& \mathrm{R}_{1}=4-\mathrm{N}\left(\mathrm{CH}_{3}\right)_{2}, \mathrm{R}_{2}=4-\mathrm{OCH}_{3},(3 \mathrm{l})
\end{aligned}
$$

Fig 1: Synthesis of novel thiazolidinone derivatives $4 a-4$ l

$4-6 \mathrm{~h}$ on a water bath. The separated solid was filtered and recrystallized from ethanol to give compounds $\mathbf{3 a}-\mathbf{3 l}$.

\section{General procedure for the synthesis of compounds 3a-3l}

To a stirred solution of compound $1(0.01 \mathrm{M})$ in ethanol $(50 \mathrm{ml})$ containing sulphuric acid $(2$ $\mathrm{ml}$ ) was added appropriate aromatic aldehyde $(0.01 \mathrm{M})$ and the mixture refluxed for $4-6 \mathrm{~h}$ on a water bath. The separated solid was filtered and recrystallized from ethanol to give compounds $\mathbf{3 a}-\mathbf{3 l}$.

\section{General procedure for the synthesis of compounds $4 a-41$}

To a stirred solution of the particular 5phenyl-N-[(substituted) phenylmethylene][1,3] thiazolo[4,3-b][1,3,4]thiadiazol-2-amine

(Schiff base 3a-l, $0.01 \mathrm{M}$ ) and thioglycollic acid $(0.01 \mathrm{M})$ in DMF $(30 \mathrm{ml})$. The reaction mixture refluxed for $6 \mathrm{~h}$ and the solid obtained after removal of the solvent was crystallized from benzene to give compounds 4a-4l.

\section{Evaluation of anti-tubercular activity}

The antimycobacterial activity of the synthesized compounds $\mathbf{3 a - I}$ and $\mathbf{4 a - I}$ were 
assessed against $M$. tuberculosis $\mathrm{H}_{37} \mathrm{Rv}$ (ATCC 2729411) using the Microplate Alamar Blue Assay (MABA) [14,15]. This methodology is non-toxic, uses thermally stable reagent, and shows good correlation with proportional and BACTEC radiometric methods [16], The activity was expressed as minimum inhibitory concentration (MIC) in $\mu \mathrm{g} / \mathrm{mL}$. The drug concentration tested were in the range $0.1-100.0 \mu \mathrm{g} / \mathrm{mL}$. A blue color in the well was interpreted as absence of bacterial growth, and pink color was scored as growth. MIC (minimal inhibition concentration) was defined as the lowest drug concentration, which prevented a color change from blue to pink. Streptomycin and pyrazinamide were used as reference standards.

\section{Data analysis}

Docking was carried out for the synthesized compounds 3a-I, and 4a-I using Hex 5.1 software Scotland, UK. Molecular docking involves the following steps using Hex 5.1 software:

1. Identify a target protein 2YES from the protein data bank.

2. Download PDB FILE (text) and save in Example Folder of Hex 5.1.

3. Draw all the ligands using ChemSketch software.

4. Generate 3-D view (SDF format) and convert it into MOL file.

5. Convert into PDB format by using Swiss PDB viewer and save it.

6. Open Hex 5.1 software, select appropriate protein and ligand and perform docking.

\section{RESULTS}

The physicochemical parameters of the synthesized compounds, including melting point, are shown in Table 1 while docking score and anti-tubercular results are listed in Tables 2 and 3, respectively. In Schiff base series compounds $\mathbf{3 f}, \mathbf{3} \mathbf{h}$ and $\mathbf{3 i}$ showed good antitubercular activity compared to standard streptomycin. In Thiazolidinone series compounds $4 \mathrm{f}$ and $4 \mathrm{i}$ showed good antitubercular activity compared to both standards pyrazinamide and streptomycin and compounds $\mathbf{4 b}, \mathbf{4 c}, \mathbf{4 e}, \mathbf{4 h}, \mathbf{4 k}, 4 \mathrm{l}$ showed similar activity compared to standard pyrazinamide but showed better than standard streptomycin. The detail spectral data of the compounds are as follows.

\section{5-phenyl-N-[(1E)-phenylmethylene][1,3] thiazolo[4,3-b][1,3,4]thiadiazol-2-amine}

(3a): FTIR (KBr) cm ${ }^{-1}$ : $3115(\mathrm{Ar} \mathrm{C}-\mathrm{H}), 2960$ $(\mathrm{C}-\mathrm{H}), 2880$ (C-H thiazole), $1590(-\mathrm{N}=\mathrm{CH})$, 702 (C-S-C); ${ }^{1} \mathrm{H}$ NMR chemical shifts at ( 400 $\left.\mathrm{MHz}_{\mathrm{CDCl}}, \delta \mathrm{ppm}\right): 11.14(\mathrm{~s}, 1 \mathrm{H}, \mathrm{CH}), 8.14$ (s, $1 \mathrm{H},-\mathrm{N}=\mathrm{CH}), 7.98(\mathrm{~s}, 1 \mathrm{H}, \mathrm{CH}), 7.30-7.68$ (m, 10H, Ar-H); MS spectrum, m/z: 324[M+1] ${ }^{+}$; Anal. Calcd for $\mathrm{C}_{17} \mathrm{H}_{13} \mathrm{~N}_{3} \mathrm{~S}_{2}$ : C (63.13), H (4.05), N (12.99). Found: C (63.11), H (4.01) and $\mathrm{N}(12.95)$.

$\mathrm{N}$-[(1E)-(2-chlorophenyl)methylene]-5phenyl[1,3]thiazolo[4,3-b][1,3,4]thiadiazol2-amine (3b): FTIR ( $\mathrm{KBr}) \mathrm{cm}^{-1}$ : 3110 (Ar C$\mathrm{H}), 2890(\mathrm{C}-\mathrm{H}), 2810$ (C-H thiazole), 1598 ($\mathrm{N}=\mathrm{CH}), 682(\mathrm{C}-\mathrm{S}-\mathrm{C}) ;{ }^{1} \mathrm{H}$ NMR chemical shifts at $\left(400 \mathrm{MHz}, \mathrm{CDCl}_{3}, \delta \mathrm{ppm}\right): 11.05(\mathrm{~s}, 1 \mathrm{H}$, $\mathrm{CH}) ; 8.04(\mathrm{~s}, 1 \mathrm{H},-\mathrm{N}=\mathrm{CH}) ; 7.86(\mathrm{~s}, 1 \mathrm{H}, \mathrm{CH})$; 7.40-7.75 (m, 9H, Ar-H); MS spectrum, m/z: $359[\mathrm{M}+1]^{+}$.

N-[(1E)-(4-chlorophenyl)methylene]-5phenyl[1,3]thiazolo[4,3-b][1,3,4]thiadiazol2-amine (3c): FTIR (KBr) cm${ }^{-1}$ : 3095 (Ar C$\mathrm{H}) ; 2930(\mathrm{C}-\mathrm{H}) ; 2820$ (C-H thiazole), 1582 ($\mathrm{N}=\mathrm{CH}), 710(\mathrm{C}-\mathrm{S}-\mathrm{C}) ;{ }^{1} \mathrm{H}$ NMR chemical shifts at $\left(400 \mathrm{MHz}, \mathrm{CDCl}_{3}, \delta \mathrm{ppm}\right): 11.10(\mathrm{~s}, 1 \mathrm{H}$, $\mathrm{CH}), 8.20(\mathrm{~s}, 1 \mathrm{H}, \mathrm{N}=\mathrm{CH}), 7.90(\mathrm{~s}, 1 \mathrm{H}, \mathrm{CH})$, 7.15-7.72 (m, 9H, Ar-H); MS spectrum, m/z: $358[\mathrm{M}+1]^{+}$.

\section{5-(4-methylphenyl)-N-[(1E)-} phenylmethylene][1,3]thiazolo[4,3-

b][1,3,4]thiadiazol-2-amine (3d): FTIR (KBr) $\mathrm{cm}^{-1}: 3157$ (Ar C-H), 2929 (C-H), 2850 (C-H thiazole), $1598(-\mathrm{N}=\mathrm{CH}), \quad 705$ (C-S-C); ${ }^{1} \mathrm{H}$ NMR chemical shifts at $\left(400 \mathrm{MHz}, \mathrm{CDCl}_{3}, \delta\right.$ ppm): $10.30(\mathrm{~s}, 1 \mathrm{H}, \mathrm{CH}), 8.37(\mathrm{~s}, 1 \mathrm{H}, \mathrm{N}=\mathrm{CH})$, $7.72(\mathrm{~s}, 1 \mathrm{H}, \mathrm{CH}), 6.86-7.52(\mathrm{~m}, 9 \mathrm{H}, \mathrm{Ar}-\mathrm{H})$, $1.45\left(\mathrm{~s}, 3 \mathrm{H}, \mathrm{CH}_{3}\right) ;{ }^{13} \mathrm{C}$ NMR $(75 \mathrm{MHz}$, DMSO- 
Malipeddi et al

Table 1: Physical data of synthesized compounds $\mathbf{3 a - 3}$ and $\mathbf{4 a - 4 I . ~}$

\begin{tabular}{|c|c|c|c|c|c|}
\hline Compd & $\mathbf{R}_{1}$ & $\mathbf{R}_{\mathbf{2}}$ & $\begin{array}{l}\text { Yield } \\
(\%)\end{array}$ & $\begin{array}{l}\text { M.P. } \\
\left({ }^{\circ} \mathrm{C}\right)\end{array}$ & Mol. formula \\
\hline $3 a$ & $-\mathrm{H}$ & $-\mathrm{H}$ & 62 & 140 & $\mathrm{C}_{17} \mathrm{H}_{13} \mathrm{~N}_{3} \mathrm{~S}_{2}$ \\
\hline $3 b$ & $-\mathrm{H}$ & $2-\mathrm{Cl}$ & 58 & 144 & $\mathrm{C}_{17} \mathrm{H}_{12} \mathrm{CIN}_{3} \mathrm{~S}_{2}$ \\
\hline $3 c$ & $-\mathrm{H}$ & $4-\mathrm{Cl}$ & 63 & 109 & $\mathrm{C}_{17} \mathrm{H}_{12} \mathrm{CIN}_{3} \mathrm{~S}_{2}$ \\
\hline $3 d$ & $4-\mathrm{CH}_{3}$ & $-\mathrm{H}$ & 71 & 114 & $\mathrm{C}_{18} \mathrm{H}_{15} \mathrm{~N}_{3} \mathrm{~S}_{2}$ \\
\hline $3 e$ & $4-\mathrm{CH}_{3}$ & $2-\mathrm{CH}_{3}$ & 78 & 132 & $\mathrm{C}_{19} \mathrm{H}_{17} \mathrm{~N}_{3} \mathrm{~S}_{2}$ \\
\hline $3 f$ & $4-\mathrm{CH}_{3}$ & $4-\mathrm{CH}_{3}$ & 76 & 156 & $\mathrm{C}_{19} \mathrm{H}_{17} \mathrm{~N}_{3} \mathrm{~S}_{2}$ \\
\hline $3 g$ & $4-\mathrm{OH}$ & $-\mathrm{H}$ & 72 & 179 & $\mathrm{C}_{17} \mathrm{H}_{13} \mathrm{~N}_{3} \mathrm{OS}_{2}$ \\
\hline $3 \mathrm{~h}$ & $4-\mathrm{OH}$ & $2-\mathrm{OH}$ & 75 & 186 & $\mathrm{C}_{17} \mathrm{H}_{13} \mathrm{~N}_{3} \mathrm{O}_{2} \mathrm{~S}_{2}$ \\
\hline $3 i$ & $4-\mathrm{OH}$ & $4-\mathrm{OH}$ & 78 & 154 & $\mathrm{C}_{17} \mathrm{H}_{13} \mathrm{~N}_{3} \mathrm{O}_{2} \mathrm{~S}_{2}$ \\
\hline $3 j$ & $4-\mathrm{N}\left(\mathrm{CH}_{3}\right)_{2}$ & $-\mathrm{H}$ & 76 & 156 & $\mathrm{C}_{19} \mathrm{H}_{18} \mathrm{~N}_{4} \mathrm{~S}_{2}$ \\
\hline $3 k$ & $4-\mathrm{N}\left(\mathrm{CH}_{3}\right)_{2}$ & $2-\mathrm{OCH}_{3}$ & 72 & 179 & $\mathrm{C}_{20} \mathrm{H}_{20} \mathrm{~N}_{4} \mathrm{OS}_{2}$ \\
\hline 31 & $4-\mathrm{N}\left(\mathrm{CH}_{3}\right)_{2}$ & $4-\mathrm{OCH}_{3}$ & 66 & 141 & $\mathrm{C}_{20} \mathrm{H}_{20} \mathrm{~N}_{4} \mathrm{OS}_{2}$ \\
\hline $4 a$ & $-\mathrm{H}$ & $-\mathrm{H}$ & 67 & 158 & $\mathrm{C}_{19} \mathrm{H}_{15} \mathrm{~N}_{3} \mathrm{OS}_{3}$ \\
\hline $4 b$ & $-\mathrm{H}$ & $2-\mathrm{Cl}$ & 61 & 163 & $\mathrm{C}_{19} \mathrm{H}_{14} \mathrm{ClN}_{3} \mathrm{OS}_{3}$ \\
\hline $4 c$ & $-\mathrm{H}$ & $4-\mathrm{Cl}$ & 65 & 129 & $\mathrm{C}_{19} \mathrm{H}_{14} \mathrm{ClN}_{3} \mathrm{OS}_{3}$ \\
\hline $4 d$ & $4-\mathrm{CH}_{3}$ & $-\mathrm{H}$ & 71 & 136 & $\mathrm{C}_{20} \mathrm{H}_{17} \mathrm{~N}_{3} \mathrm{OS}_{3}$ \\
\hline $4 e$ & $4-\mathrm{CH}_{3}$ & $2-\mathrm{CH}_{3}$ & 73 & 146 & $\mathrm{C}_{21} \mathrm{H}_{19} \mathrm{~N}_{3} \mathrm{OS}_{3}$ \\
\hline $4 f$ & $4-\mathrm{CH}_{3}$ & $4-\mathrm{CH}_{3}$ & 74 & 156 & $\mathrm{C}_{21} \mathrm{H}_{19} \mathrm{~N}_{3} \mathrm{OS}_{3}$ \\
\hline $4 g$ & $4-\mathrm{OH}$ & $-\mathrm{H}$ & 71 & 167 & $\mathrm{C}_{19} \mathrm{H}_{15} \mathrm{~N}_{3} \mathrm{O}_{2} \mathrm{~S}_{3}$ \\
\hline $4 \mathrm{~h}$ & $4-\mathrm{OH}$ & $2-\mathrm{OH}$ & 73 & 123 & $\mathrm{C}_{19} \mathrm{H}_{15} \mathrm{~N}_{3} \mathrm{O}_{3} \mathrm{~S}_{3}$ \\
\hline $4 i$ & $4-\mathrm{OH}$ & $4-\mathrm{OH}$ & 75 & 167 & $\mathrm{C}_{19} \mathrm{H}_{15} \mathrm{~N}_{3} \mathrm{O}_{3} \mathrm{~S}_{3}$ \\
\hline $4 j$ & $4-\mathrm{N}\left(\mathrm{CH}_{3}\right)_{2}$ & $-\mathrm{H}$ & 70 & 189 & $\mathrm{C}_{21} \mathrm{H}_{20} \mathrm{~N}_{4} \mathrm{OS}_{3}$ \\
\hline $4 k$ & $4-\mathrm{N}\left(\mathrm{CH}_{3}\right)_{2}$ & $2-\mathrm{OCH}_{3}$ & 71 & 134 & $\mathrm{C}_{22} \mathrm{H}_{22} \mathrm{~N}_{4} \mathrm{O}_{2} \mathrm{~S}_{3}$ \\
\hline 41 & $4-\mathrm{N}\left(\mathrm{CH}_{3}\right)_{2}$ & $4-\mathrm{OCH}_{3}$ & 67 & 156 & $\mathrm{C}_{22} \mathrm{H}_{22} \mathrm{~N}_{4} \mathrm{O}_{2} \mathrm{~S}_{3}$ \\
\hline
\end{tabular}

Table 2: Antitubercular activity and docking score of compounds 3a-3I

\begin{tabular}{|c|c|c|c|c|c|}
\hline Compound & $\mathbf{R}_{1}$ & $\mathbf{R}_{\mathbf{2}}$ & $\begin{array}{l}\text { MIC } \\
(\mu \mathrm{g} / \mathrm{mL})\end{array}$ & PDB code & $\begin{array}{l}\text { E score } \\
(\mathrm{kJ} / \mathrm{mol})\end{array}$ \\
\hline $3 a$ & $-\mathrm{H}$ & $-\mathrm{H}$ & 12.5 & 2YES & -139.92 \\
\hline $3 b$ & $-H$ & $2-\mathrm{Cl}$ & 6.25 & 2YES & -144.60 \\
\hline $3 c$ & $-\mathrm{H}$ & $4-\mathrm{Cl}$ & 6.25 & 2YES & -144.60 \\
\hline $3 d$ & $4-\mathrm{CH}_{3}$ & $-\mathrm{H}$ & 12.5 & 2YES & -143.75 \\
\hline $3 e$ & $4-\mathrm{CH}_{3}$ & $2-\mathrm{CH}_{3}$ & 6.25 & 2YES & -145.74 \\
\hline $3 f$ & $4-\mathrm{CH}_{3}$ & 4- $\mathrm{CH}_{3}$ & 3.125 & 2YES & -145.74 \\
\hline $3 g$ & $4-\mathrm{OH}$ & $-\mathrm{H}$ & 12.5 & 2YES & -138.60 \\
\hline $3 h$ & $4-\mathrm{OH}$ & $2-\mathrm{OH}$ & 3.125 & 2YES & -144.07 \\
\hline $3 i$ & $4-\mathrm{OH}$ & $4-\mathrm{OH}$ & 3.125 & 2YES & -144.07 \\
\hline $3 \mathrm{j}$ & $4-\mathrm{N}\left(\mathrm{CH}_{3}\right)_{2}$ & $-\mathrm{H}$ & 6.25 & 2YES & -145.62 \\
\hline $3 k$ & $4-\mathrm{N}\left(\mathrm{CH}_{3}\right)_{2}$ & $2-\mathrm{OCH}_{3}$ & 6.25 & 2YES & -147.61 \\
\hline 31 & $4-\mathrm{N}\left(\mathrm{CH}_{3}\right)_{2}$ & $4-\mathrm{OCH}_{3}$ & 6.25 & 2YES & -147.61 \\
\hline Streptomycin & - & - & 6.25 & 2YES & -125.43 \\
\hline Pyrazinamide & - & - & 3.12 & 2YES & -123.07 \\
\hline
\end{tabular}


Malipeddi et al

Table 3: Antitubercular activity and docking score of compounds 4a-4I

\begin{tabular}{llllll}
\hline Compound & $\mathbf{R}_{\mathbf{1}}$ & $\mathbf{R}_{\mathbf{2}}$ & $\begin{array}{l}\mathbf{M I C} \\
(\boldsymbol{\mu g} / \mathbf{m L})\end{array}$ & PDB code & $\begin{array}{l}\mathbf{E} \text { score } \\
(\mathbf{k J} / \mathbf{m o l})\end{array}$ \\
\hline $4 \mathrm{a}$ & $-\mathrm{H}$ & $-\mathrm{H}$ & 6.25 & $2 \mathrm{YES}$ & -147.51 \\
$4 \mathrm{~b}$ & $-\mathrm{H}$ & $2-\mathrm{Cl}$ & 3.12 & $2 \mathrm{YES}$ & -147.31 \\
$4 \mathrm{c}$ & $-\mathrm{H}$ & $4-\mathrm{Cl}$ & 3.12 & $2 \mathrm{YES}$ & -147.31 \\
$4 \mathrm{~d}$ & $4-\mathrm{CH}_{3}$ & $-\mathrm{H}$ & 6.25 & $2 \mathrm{YES}$ & -147.53 \\
$4 \mathrm{e}$ & $4-\mathrm{CH}_{3}$ & $2-\mathrm{CH}_{3}$ & 3.12 & $2 \mathrm{YES}$ & -146.04 \\
$4 \mathrm{f}$ & $4-\mathrm{CH}_{3}$ & $4-\mathrm{CH}_{3}$ & 1.6 & $2 \mathrm{YES}$ & -146.04 \\
$4 \mathrm{~g}$ & $4-\mathrm{OH}$ & $-\mathrm{H}$ & 6.25 & $2 \mathrm{YES}$ & -146.99 \\
$4 \mathrm{~h}$ & $4-\mathrm{OH}$ & $2-\mathrm{OH}$ & 3.12 & $2 \mathrm{YES}$ & -147.00 \\
$4 \mathrm{i}$ & $4-\mathrm{OH}$ & $4-\mathrm{OH}$ & 1.6 & $2 \mathrm{YES}$ & -147.00 \\
$4 \mathrm{j}$ & $4-\mathrm{N}\left(\mathrm{CH}_{3}\right)_{2}$ & $-\mathrm{H}$ & 6.25 & $2 \mathrm{YES}$ & -146.01 \\
$4 \mathrm{k}$ & $4-\mathrm{N}\left(\mathrm{CH}_{3}\right)_{2}$ & $2-\mathrm{OCH}_{3}$ & 3.12 & $2 \mathrm{YES}$ & -146.62 \\
$4 \mathrm{l}$ & $4-\mathrm{N}\left(\mathrm{CH}_{3}\right)_{2}$ & $4-\mathrm{OCH}_{3}$ & 3.12 & $2 \mathrm{YES}$ & -146.62 \\
Streptomycin & - & - & 6.25 & $2 \mathrm{YES}$ & -125.43 \\
Pyrazinamide & - & - & 3.12 & $2 \mathrm{YES}$ & -123.07 \\
\hline
\end{tabular}

$\left.d_{6}\right): \delta 32.65,43.74,130.60,134.86,140.93$, $144.85,150.86,153.55,160.17,160.68$, 163.23, 164.40;MS spectrum, m/z: 338 [M+1] ${ }^{+}$; Anal. Calcd for $\mathrm{C}_{18} \mathrm{H}_{15} \mathrm{~N}_{3} \mathrm{~S}_{2}$ : C (64.06), H (4.48), N (12.45). Found: C (64.01), H (4.44) and $\mathrm{N}(12.39)$.

\section{5-(4-methylphenyl)-N-[(1E)-(2-}

methylphenyl)methylene][1,3]thiazolo[4,3b][1,3,4] thiadiazol-2-amine (3e): FTIR $(\mathrm{KBr}) \mathrm{cm}^{-1}$ : 3108 (Ar C-H), 2950(C-H), 2870 (C-H thiazole), 1585 (-N=CH), 712 (C-S-C); ${ }^{1} \mathrm{H}$ NMR chemical shifts at $\left(400 \mathrm{MHz}, \mathrm{CDCl}_{3}\right.$, $\delta \mathrm{ppm}): 10.16(\mathrm{~s}, 1 \mathrm{H}, \mathrm{CH}), 8.39(\mathrm{~s}, 1 \mathrm{H}$, $\mathrm{N}=\mathrm{CH}), 7.86(\mathrm{~s}, 1 \mathrm{H}, \mathrm{CH}), 7.25-7.63(\mathrm{~m}, 8 \mathrm{H}$, $\mathrm{Ar}-\mathrm{H}), 2.45\left(\mathrm{~s}, 6 \mathrm{H}, \mathrm{CH}_{3}\right)$; MS spectrum, m/z: $353[\mathrm{M}+1]^{+}$.

\section{5-(4-methylphenyl)-N-[(1E)-(4-} methylphenyl)methylene][1,3]thiazolo[4,3b] $[1,3,4]$ thiadiazol-2-amine (3f): FTIR (KBr) $\mathrm{cm}^{-1}$ : 3005 (Ar C-H), $2890(\mathrm{C}-\mathrm{H}), 2750$ (C-H thiazole), $1583(-\mathrm{N}=\mathrm{CH}), 712(\mathrm{C}-\mathrm{S}-\mathrm{C}) ;{ }^{1} \mathrm{H}$ NMR chemical shifts at $\left(400 \mathrm{MHz}, \mathrm{CDCl}_{3}, \delta\right.$ ppm): 10.14 (s, $1 \mathrm{H}, \mathrm{CH}), 8.36(\mathrm{~s}, 1 \mathrm{H}, \mathrm{N}=\mathrm{CH})$, $7.81(\mathrm{~s}, 1 \mathrm{H}, \mathrm{CH}), 7.22-7.68(\mathrm{~m}, 8 \mathrm{H}, \mathrm{Ar}-\mathrm{H})$, $2.35\left(\mathrm{~s}, 6 \mathrm{H}, \mathrm{CH}_{3}\right) ; \mathrm{MS}$ spectrum, $\mathrm{m} / \mathrm{z}: 352$ $[\mathrm{M}+1]^{+}$.
4-(2-\{[(1E)-phenylmethylene]amino $\}[1,3]$ thiazolo[4,3-b][1,3,4] thiadiazol-5-yl)phenol (3g): FTIR (KBr) cm ${ }^{-1}: 3545(\mathrm{O}-\mathrm{H}), 3005$ (ArC-H), $2955(\mathrm{C}-\mathrm{H}), 2875$ (C-H thiazole), $1515 \quad(-\mathrm{N}=\mathrm{CH}), \quad 695 \quad(\mathrm{C}-\mathrm{S}-\mathrm{C}) ;{ }^{1} \mathrm{H} \quad \mathrm{NMR}$ chemical shifts at $\left(400 \mathrm{MHz}, \mathrm{CDCl}_{3}, \delta \mathrm{ppm}\right)$ : $9.63(\mathrm{~s}, 1 \mathrm{H}, \mathrm{CH}), 7.83(\mathrm{~s}, 1 \mathrm{H}, \mathrm{CH}), 8.19(\mathrm{~s}$, $1 \mathrm{H}, \mathrm{N}=\mathrm{CH}), 7.18-7.34(\mathrm{~m}, 9 \mathrm{H}, \mathrm{Ar}-\mathrm{H}), 5.28(\mathrm{~s}$, $1 \mathrm{H}, \mathrm{OH}) ; \mathrm{MS}$ spectrum, m/z: $341[\mathrm{M}+1]^{+}$.

\section{2-[(E)-\{[5-(4-hydroxyphenyl)[1,3]thiazolo} [4,3-b][1,3,4]thiadiazol-2-yl]imino\}methyl] phenol (3h): FTIR (KBr) cm $\mathrm{cm}^{-1}$ : $3515(\mathrm{O}-\mathrm{H})$, 3005 (Ar C-H), 2965 (C-H), 2885 (C-H thiazole), $1550(-\mathrm{N}=\mathrm{CH}), 702$ (C-S-C); ${ }^{1} \mathrm{H}$ NMR chemical shifts at $\left(400 \mathrm{MHz}, \mathrm{CDCl}_{3}, \delta\right.$ ppm): $9.82(\mathrm{~s}, 1 \mathrm{H}, \mathrm{CH}), 7.95(\mathrm{~s}, 1 \mathrm{H}, \mathrm{CH})$, $8.22(\mathrm{~s}, 1 \mathrm{H}, \mathrm{N}=\mathrm{CH}), 7.05-7.45(\mathrm{~m}, 8 \mathrm{H}, \mathrm{Ar}-\mathrm{H})$, $5.20(\mathrm{~s}, 2 \mathrm{H}, \mathrm{OH}) ; \mathrm{MS}$ spectrum, m/z: 356 $[\mathrm{M}+1]^{+}$.

\section{4-(2-\{[(1E)-(4-hydroxyphenyl)methylene]} amino\}[1,3]thiazolo[4,3-b][1,3,4]thiadiazol5-yl)phenol (3i): FTIR (KBr) cm $\mathrm{cm}^{-1}$ : 3530 (O$\mathrm{H}), 3005$ (ArC-H), $2955(\mathrm{C}-\mathrm{H}), 2875(\mathrm{C}-\mathrm{H}$ thiazole), 1535 (-N=CH), 780 (C-S-C); ${ }^{1} \mathrm{H}$ NMR chemical shifts at $\left(400 \mathrm{MHz}, \mathrm{CDCl}_{3}, \delta\right.$ ppm): $9.85(\mathrm{~s}, 1 \mathrm{H}, \mathrm{CH}), 7.98(\mathrm{~s}, 1 \mathrm{H}, \mathrm{CH})$, $8.30(\mathrm{~s}, 1 \mathrm{H}, \mathrm{N}=\mathrm{CH}), 7.10-7.38(\mathrm{~m}, 8 \mathrm{H}, \mathrm{Ar}-\mathrm{H})$, 
5.18 (s, 2H, OH); MS spectrum, m/z: 357 $[\mathrm{M}+1]^{+}$.

\section{5-[4-(dimethylamino)phenyl]-N-[(1E)-}

phenylmethylene][1,3]thiazolo[4,3-b][1,3,4] thiadiazol-2-amine (3j): FTIR ( $\mathrm{KBr}) \mathrm{cm}^{-1}$ : 3111 (Ar C-H), 2962 (C-H), 2875 (C-H thiazole), $1583(-\mathrm{N}=\mathrm{CH}), 714$ (C-S-C); ${ }^{1} \mathrm{H}$ NMR chemical shifts at $\left(400 \mathrm{MHz}, \mathrm{CDCl}_{3}, \delta\right.$ ppm): $9.86(\mathrm{~s}, 1 \mathrm{H}, \mathrm{CH}), 9.39(\mathrm{~s}, 1 \mathrm{H}, \mathrm{N}=\mathrm{CH})$, $6.34(\mathrm{~s}, 1 \mathrm{H}, \mathrm{CH}), 7.17-7.72(\mathrm{~m}, 9 \mathrm{H}, \mathrm{Ar}-\mathrm{H})$, $1.42-1.47\left(\mathrm{~s}, 6 \mathrm{H}, \mathrm{CH}_{3}\right)$; $\mathrm{MS}$ spectrum, $\mathrm{m} / \mathrm{z}$ : $368[\mathrm{M}+1]^{+}$.

\section{5-[4-(dimethylamino)phenyl]-N-[(1E)-(2- methoxyphenyl)methylene][1,3]thiazolo} [4,3-b] [1,3,4]thiadiazol -2-amine (3k): FTIR $(\mathrm{KBr}) \mathrm{cm}^{-1}: 3120$ (Ar C-H), $2965(\mathrm{C}-\mathrm{H}), 2892$ (C-H thiazole), $1585(-\mathrm{N}=\mathrm{CH}), 718(\mathrm{C}-\mathrm{S}-\mathrm{C})$; H NMR chemical shifts at $\left(400 \mathrm{MHz}, \mathrm{CDCl}_{3}\right.$, $\delta \mathrm{ppm}): 9.92(\mathrm{~s}, 1 \mathrm{H}, \mathrm{CH}), 9.28(\mathrm{~s}, 1 \mathrm{H}, \mathrm{N}=\mathrm{CH})$, $6.37(\mathrm{~s}, 1 \mathrm{H}, \mathrm{CH}), 7.80-7.95(\mathrm{~m}, 8 \mathrm{H}, \mathrm{Ar}-\mathrm{H})$, $3.15\left(\mathrm{~s}, 3 \mathrm{H},-\mathrm{OCH}_{3}\right), 1.42-1.47\left(\mathrm{~s}, 6 \mathrm{H}, \mathrm{CH}_{3}\right)$; MS spectrum, m/z: $398[\mathrm{M}+1]^{+}$.

\section{5-[4-(dimethylamino)phenyl]-N-[(1E)-(4- methoxyphenyl)methylene][1,3]thiazolo} [4,3-b][1,3,4] thiadiazol-2-amine (3I): FTIR (KBr) cm ${ }^{-1}$ : 3122 (Ar C-H), $2972(\mathrm{C}-\mathrm{H}), 2892$ (C-H thiazole), $1594(-\mathrm{N}=\mathrm{CH}), 722(\mathrm{C}-\mathrm{S}-\mathrm{C})$; $\mathrm{H}$ NMR chemical shifts at $\left(400 \mathrm{MHz}, \mathrm{CDCl}_{3}\right.$, $\delta \mathrm{ppm}): 9.80(\mathrm{~s}, 1 \mathrm{H}, \mathrm{CH}), 9.30(\mathrm{~s}, 1 \mathrm{H}, \mathrm{N}=\mathrm{CH})$, $6.43(\mathrm{~s}, 1 \mathrm{H}, \mathrm{CH}), 7.75-7.98(\mathrm{~m}, 8 \mathrm{H}, \mathrm{Ar}-\mathrm{H})$, $3.52\left(\mathrm{~s}, 3 \mathrm{H},-\mathrm{OCH}_{3}\right), 1.52-1.58\left(\mathrm{~s}, 6 \mathrm{H}, \mathrm{CH}_{3}\right)$; MS spectrum, m/z: $397[\mathrm{M}+1]^{+}$.

\section{2-phenyl-3-(5-phenyl[1,3]thiazolo[4,3-} b] [1,3,4]thiadiazol-2-yl)-1,3-thiazolidin-4one(4a): FTIR (KBr) cm${ }^{-1}$ : $3121(\mathrm{Ar} \mathrm{C}-\mathrm{H})$; 2972 (C-H); 2877 (C-H thiazole); 1692 ($\mathrm{C}=\mathrm{O}), 705$ (C-S-C). ${ }^{1} \mathrm{H}$ NMR chemical shifts at $\left(400 \mathrm{MHz}, \mathrm{CDCl}_{3}, \delta \mathrm{ppm}\right): 11.21(\mathrm{~s}, 1 \mathrm{H}$, $\mathrm{CH}) ; 7.82(\mathrm{~s}, 1 \mathrm{H}, \mathrm{CH}) ; 7.28-7.58(\mathrm{~m}, 10 \mathrm{H}, \mathrm{Ar}-$ $\mathrm{H}) ; 4.11(\mathrm{~s}, 1 \mathrm{H},-\mathrm{N}-\mathrm{CH}), 4.08\left(\mathrm{~s}, 2 \mathrm{H}, \mathrm{SCH}_{2}\right)$; MS spectrum, $\mathrm{m} / \mathrm{z}$ : $399[\mathrm{M}+1]^{+}$; Anal. Calcd. for $\mathrm{C}_{19} \mathrm{H}_{15} \mathrm{~N}_{3} \mathrm{OS}_{3}$ : $\mathrm{C}$ (57.40), $\mathrm{H}$ (3.80), $\mathrm{N}$ (10.57). Found: $\mathrm{C}$ (57.35), $\mathrm{H}(3.74)$ and $\mathrm{N}$ (10.49). 2-(2-chlorophenyl)-3-(5-phenyl[1,3] thiazolo[4,3-b][1,3,4]thiadiazol-2-yl)-1,3thiazolidin-4-one (4b): FTIR $(\mathrm{KBr}) \mathrm{cm}^{-1}$ : 3112 (Ar C-H); 2950 (C-H); 2870 (C-H thiazole); 1685 (-C=O), 703 (C-S-C), 761 (-C$\mathrm{Cl}) .{ }^{1} \mathrm{H}$ NMR chemical shifts at $(400 \mathrm{MHz}$, $\mathrm{CDCl}_{3}, \delta \mathrm{ppm}$ ): $11.11(\mathrm{~s}, 1 \mathrm{H}, \mathrm{CH}) ; 7.92$ (s, $1 \mathrm{H}, \mathrm{CH})$; 7.35-7.75 (m, 9H, Ar-H) 4.12 (s, 1H, $-\mathrm{N}-\mathrm{CH}), 4.17\left(\mathrm{~s}, 2 \mathrm{H}, \mathrm{SCH}_{2}\right) ; \mathrm{MS}$ spectrum, $\mathrm{m} / \mathrm{z}: 432[\mathrm{M}+1]^{+}$.

\section{2-(4-chlorophenyl)-3-(5-phenyl[1,3]}

thiazolo[4,3-b][1,3,4]thiadiazol-2-yl)-1,3thiazolidin-4-one (4c): FTIR (KBr) cm$~^{-1}$ : 3115 (Ar C-H); 2972 (C-H); 2869 (C-H thiazole); 1674 (-C=O), 710 (C-S-C), 755 (-C$\mathrm{Cl}) .{ }^{1} \mathrm{H}$ NMR chemical shifts at $(400 \mathrm{MHz}$, $\left.\mathrm{CDCl}_{3}, \delta \mathrm{ppm}\right): 11.09(\mathrm{~s}, 1 \mathrm{H}, \mathrm{CH}) ; 7.76$ (s, $1 \mathrm{H}, \mathrm{CH}) ; 7.30-7.70(\mathrm{~m}, 9 \mathrm{H}, \mathrm{Ar}-\mathrm{H}) ; 4.10$ (s, $1 \mathrm{H}, \quad-\mathrm{N}-\mathrm{CH}), \quad 4.18\left(\mathrm{~s}, 2 \mathrm{H}, \quad \mathrm{SCH}_{2}\right) ; \mathrm{MS}$ spectrum, $m / z: 433[\mathrm{M}+1]^{+}$.

\section{3-[5-(4-methylphenyl)[1,3]thiazolo[4,3-}

\section{b] $[1,3,4]$ thiadiazol-2-yl]-2-phenyl-1,3-}

thiazolidin-4-one (4d): FTIR (KBr) cm${ }^{-1}$ : 3153 (Ar C-H); 2928 (C-H); 2358 (C-H thiazole); 1693 (-C=O), 723 (C-S-C). ${ }^{1} \mathrm{H}$ NMR chemical shifts at $\left(400 \mathrm{MHz}, \mathrm{CDCl}_{3}, \delta \mathrm{ppm}\right)$ : 10.05 (s, 1H, CH); 7.87 (s, 1H, CH); 7.21$7.24(\mathrm{~m}, 9 \mathrm{H}, \mathrm{Ar}-\mathrm{H}) ; 2.84\left(\mathrm{~s}, 3 \mathrm{H}, \mathrm{CH}_{3}\right) ; 4.44$ (s, $1 \mathrm{H},-\mathrm{N}-\mathrm{CH}), 4.72$ (s, $\left.2 \mathrm{H}, \mathrm{SCH}_{2}\right) ;{ }^{13} \mathrm{C}$ NMR $(75$ $\left.\mathrm{MHz}, \mathrm{DMSO}_{-} \mathrm{d}_{6}\right): \delta 28.02,28.27,28.32$, $32.87, \quad 37.26, \quad 128.93, \quad 130.60, \quad 132.44$, $137.26,140.97,144.65,153.55,160.17$, 160.68, 163.33, 164.43, 177.97; MS spectrum, MS spectrum, $\mathrm{m} / \mathrm{z}: 412[\mathrm{M}+1]^{+}$; Anal. Calcd. for $\mathrm{C}_{20} \mathrm{H}_{17} \mathrm{~N}_{3} \mathrm{OS}_{3}$ : C (58.37), H (4.16), N (10.21). Found: C (58.31), H (4.14) and $\mathrm{N}(10.14)$.

\section{2-(2-methylphenyl)-3-[5-(4-}

\section{methylphenyl)[1,3]thiazolo[4,3-}

b][1,3,4]thiadiazol-2-yl]-1,3-thiazolidin-4one (4e): FTIR (KBr) cm$~^{-1}$ : $3110(\mathrm{Ar} \mathrm{C}-\mathrm{H})$; 2960 (C-H); 2880 (C-H thiazole); 1685 ($\mathrm{C}=\mathrm{O}), 669$ (C-S-C). ${ }^{1} \mathrm{H}$ NMR chemical shifts at $\left(400 \mathrm{MHz}, \mathrm{CDCl}_{3}, \delta \mathrm{ppm}\right): 10.16(\mathrm{~s}, 1 \mathrm{H}$, $\mathrm{CH}) ; 7.86(\mathrm{~s}, 1 \mathrm{H}, \mathrm{CH}) ; 7.32-7.63(\mathrm{~m}, 8 \mathrm{H}, \mathrm{Ar}-$ $\mathrm{H}) ; 2.74\left(\mathrm{~s}, 6 \mathrm{H}, \mathrm{CH}_{3}\right) ; 4.06(\mathrm{~s}, 1 \mathrm{H},-\mathrm{N}-\mathrm{CH})$, 
$4.19\left(\mathrm{~s}, 2 \mathrm{H}, \mathrm{SCH}_{2}\right) ; \quad \mathrm{MS}$ spectrum, MS spectrum, $m / z: 426[\mathrm{M}+1]^{+}$.

2-(4-methylphenyl)-3-[5-(4methylphenyl) $[1,3]$ thiazolo $[4,3-$

b][1,3,4]thiadiazol-2-yl]-1,3-thiazolidin-4one (4f): FTIR (KBr) cm ch $^{-1} 3153(\mathrm{Ar} \mathrm{C}-\mathrm{H})$; 2980 (C-H); 2895 (C-H thiazole); 1748 ($\mathrm{C}=\mathrm{O}), 713$ (C-S-C). ${ }^{1} \mathrm{H}$ NMR chemical shifts at $\left(400 \mathrm{MHz}, \mathrm{CDCl}_{3}, \delta \mathrm{ppm}\right): 10.15(\mathrm{~s}, 1 \mathrm{H}$, $\mathrm{CH}) ; 7.81(\mathrm{~s}, 1 \mathrm{H}, \mathrm{CH}) ; 7.32-7.48(\mathrm{~m}, 8 \mathrm{H}, \mathrm{Ar}-$ $\mathrm{H}) ; 2.24\left(\mathrm{~s}, 6 \mathrm{H}, \mathrm{CH}_{3}\right) ; 4.12(\mathrm{~s}, 1 \mathrm{H},-\mathrm{N}-\mathrm{CH})$, $4.30\left(\mathrm{~s}, 2 \mathrm{H}, \mathrm{SCH}_{2}\right) ; \quad \mathrm{MS}$ spectrum, MS spectrum, $m / z: 427[\mathrm{M}+1]^{+}$.

3-[5-(4-hydroxyphenyl)[1,3]thiazolo[4,3b] $[1,3,4]$ thiadiazol-2-yl]-2-phenyl-1,3-

thiazolidin-4-one (4g): FTIR (KBr) cm$~^{-1}$ : 3545 (O-H); 3005 (Ar C-H); 2955 (C-H); 2875 (C-H thiazole); $1738(-\mathrm{C}=\mathrm{O}), 675(\mathrm{C}-\mathrm{S}-\mathrm{C}) .{ }^{1} \mathrm{H}$ NMR chemical shifts at $\left(400 \mathrm{MHz}, \mathrm{CDCl}_{3}, \delta\right.$ ppm): $9.63(\mathrm{~s}, 1 \mathrm{H}, \mathrm{CH}) ; 7.83(\mathrm{~s}, 1 \mathrm{H}, \mathrm{CH})$; 7.18-7.34 (m, 9H, Ar-H); ; $5.28(\mathrm{~s}, 1 \mathrm{H}, \mathrm{OH})$; $4.15(\mathrm{~s}, 1 \mathrm{H},-\mathrm{N}-\mathrm{CH}), 4.19$ (s, $\left.2 \mathrm{H}, \mathrm{SCH}_{2}\right) ; \mathrm{MS}$ spectrum, $m / z: 415[\mathrm{M}+1]^{+}$.

2-(2-hydroxyphenyl)-3-[5-(4hydroxyphenyl)[1,3]thiazolo[4,3-

b][1,3,4]thiadiazol-2-yl]-1,3-thiazolidin-4one (4h): FTIR (KBr) cm cm $^{-1} 3515(\mathrm{O}-\mathrm{H}) ; 3005$ (Ar C-H); $2955(\mathrm{C}-\mathrm{H}) ; 2875$ (C-H thiazole); 1750 (-C=O), 705 (C-S-C). ${ }^{1} \mathrm{H}$ NMR chemical shifts at $\left(400 \mathrm{MHz}, \mathrm{CDCl}_{3}, \delta \mathrm{ppm}\right): 9.63$ (s, $1 \mathrm{H}, \mathrm{CH}) ; 7.83(\mathrm{~s}, 1 \mathrm{H}, \mathrm{CH}) ; 7.28-7.44(\mathrm{~m}, 8 \mathrm{H}$, $\operatorname{Ar}-\mathrm{H}) ; 5.20(\mathrm{~s}, 2 \mathrm{H}, \mathrm{OH}) ; 4.10(\mathrm{~s}, 1 \mathrm{H},-\mathrm{N}-\mathrm{CH})$, $4.17\left(\mathrm{~s}, 2 \mathrm{H}, \mathrm{SCH}_{2}\right)$; MS spectrum, $\mathrm{m} / \mathrm{z}: 430$ $[\mathrm{M}+1]^{+}$.

\section{2-(4-hydroxyphenyl)-3-[5-(4-}

hydroxyphenyl) $[1,3]$ thiazolo[4,3-

b][1,3,4] thiadiazol-2-yl]-1,3-thiazolidin-4-

one (4i): FTIR (KBr) cm ${ }^{-1}: 3530(\mathrm{O}-\mathrm{H}) ; 3005$ (Ar C-H); 2955 (C-H); 2875 (C-H thiazole); $1738(-\mathrm{C}=\mathrm{O}), 715$ (C-S-C). ${ }^{1} \mathrm{H}$ NMR chemical shifts at $\left(400 \mathrm{MHz}, \mathrm{CDCl}_{3}, \delta \mathrm{ppm}\right): 9.63$ (s, $1 \mathrm{H}, \mathrm{CH}) ; 7.83(\mathrm{~s}, 1 \mathrm{H}, \mathrm{CH}) ; 7.32-7.54(\mathrm{~m}, 8 \mathrm{H}$, $\mathrm{Ar}-\mathrm{H}) ; 5.28$ (s, 2H, OH); $4.11(\mathrm{~s}, 1 \mathrm{H},-\mathrm{N}-\mathrm{CH})$, $4.23\left(\mathrm{~s}, 2 \mathrm{H}, \mathrm{SCH}_{2}\right) ; \mathrm{MS}$ spectrum, $\mathrm{m} / \mathrm{z}: 431$ $[\mathrm{M}+1]^{+}$. 3-\{5-[4-(dimethylamino)phenyl][1,3] thiazolo $[4,3-b][1,3,4]$ thiadiazol-2-yl $\}-2-$ phenyl-1,3-thiazolidin-4-one (4j): FTIR $(\mathrm{KBr}) \mathrm{cm}^{-1}$ : 3105 (Ar C-H); 2945 (C-H); 2785 (C-H thiazole); 1695 (-C=O), 703 (C-S-C). ${ }^{1} \mathrm{H}$ NMR chemical shifts at $\left(400 \mathrm{MHz}, \mathrm{CDCl}_{3}, \delta\right.$ ppm): $9.84(\mathrm{~s}, 1 \mathrm{H}, \mathrm{CH}) ; 6.36(\mathrm{~s}, 1 \mathrm{H}, \mathrm{CH})$; 7.39-7.72 (m, 9H, Ar-H); 1.42-1.47(s, 6H, $\left.\mathrm{CH}_{3}\right) ; 4.18(\mathrm{~s}, 1 \mathrm{H},-\mathrm{N}-\mathrm{CH}), 4.25$ (s, 2H, $\left.\mathrm{SCH}_{2}\right)$; MS spectrum, $\mathrm{m} / \mathrm{z}: 442[\mathrm{M}+1]^{+}$.

\section{2-(2-methoxyphenyl)-3-\{5-[4-}

(dimethylamino)phenyl][1,3]thiazolo[4,3-

b] $[1,3,4]$ thiadiazol-2-yl\}-1,3-thiazolidin-4one(4k): FTIR (KBr) cm$~^{-1}$ : 3095 (Ar C-H); 2895 (C-H); 2760 (C-H thiazole); 1675 ($\mathrm{C}=\mathrm{O}), 675$ (C-S-C). ${ }^{1} \mathrm{H}$ NMR chemical shifts at $\left(400 \mathrm{MHz}, \mathrm{CDCl}_{3}, \delta \mathrm{ppm}\right): 9.77(\mathrm{~s}, 1 \mathrm{H}$, $\mathrm{CH}) ; 6.45(\mathrm{~s}, 1 \mathrm{H}, \mathrm{CH}) ; 7.80-7.95(\mathrm{~m}, 8 \mathrm{H}, \mathrm{Ar}-$ $\mathrm{H}) ; 3.15\left(\mathrm{~s}, 3 \mathrm{H},-\mathrm{OCH}_{3}\right) ; 1.42-1.47(\mathrm{~s}, 6 \mathrm{H}$, $\left.\mathrm{CH}_{3}\right) ; 4.13(\mathrm{~s}, 1 \mathrm{H},-\mathrm{N}-\mathrm{CH}), 4.21(\mathrm{~s}, 2 \mathrm{H}$, $\mathrm{SCH}_{2}$ ); MS spectrum, $\mathrm{m} / \mathrm{z}: 472[\mathrm{M}+1]^{+}$.

\section{2-(4-methoxyphenyl)-3-\{5-[4-}

(dimethylamino)phenyl][1,3]thiazolo[4,3-

b] $[1,3,4]$ thiadiazol-2-yl\}-1,3-thiazolidin-4one (4I): FTIR (KBr) cm ${ }^{-1}: 3105(\mathrm{Ar} \mathrm{C}-\mathrm{H})$; 2925 (C-H); 2880 (C-H thiazole); 1666 ($\mathrm{C}=\mathrm{O}), 708$ (C-S-C). ${ }^{1} \mathrm{H}$ NMR chemical shifts at $\left(400 \mathrm{MHz}, \mathrm{CDCl}_{3}, \delta \mathrm{ppm}\right): 9.67(\mathrm{~s}, 1 \mathrm{H}$, $\mathrm{CH}) ; 6.49(\mathrm{~s}, 1 \mathrm{H}, \mathrm{CH}) ; 7.66-7.89(\mathrm{~m}, 8 \mathrm{H}, \mathrm{Ar}-$ $\mathrm{H}) ; 3.55\left(\mathrm{~s}, 3 \mathrm{H},-\mathrm{OCH}_{3}\right) ; .1 .40-1.49(\mathrm{~s}, 6 \mathrm{H}$, $\left.\mathrm{CH}_{3}\right) ; 4.05(\mathrm{~s}, 1 \mathrm{H},-\mathrm{N}-\mathrm{CH}), 4.19$ (s, 2H, $\mathrm{SCH}_{2}$ ); MS spectrum, $\mathrm{m} / \mathrm{z}$ : $473[\mathrm{M}+1]^{+}$.

\section{Docking}

The synthesized compounds showed good docking score compared to the standard drugs, streptomycin and pyrazinamide. Tabulation of ligands docking score for 3a-I and 4a-I are shown in Tables 2 and 3, respectively.

\section{Anti-tubercular activity}

The synthesized compounds showed higher in vitro activity than the standard drugs, streptomycin and pyrazinamide. In the Schiff base series $\mathbf{3 a}-\mathbf{I}$, compounds $\mathbf{3 f}, \mathbf{3 h}$ and $\mathbf{3 i}$ 
showed good antitubercular activity compared to streptomycin. In the Thiazolidinone series $\mathbf{4 a - I}$, compounds $\mathbf{4 f}$ and 4i showed higher antitubercular activity than both pyrazinamide and streptomycin; compounds 4b, 4c, 4e, 4h, 4k, 4l showed similar activity to pyrazinamide but they all exhibited greater activity than streptomycin.

\section{DISCUSSION}

FTIR, ${ }^{1} \mathrm{H}-\mathrm{NMR},{ }^{13} \mathrm{C}-\mathrm{NMR}$ and mass spectra are in agreement with the proposed structures. All synthesized compounds showed good docking score compared to the standard drugs while the thiazolidinone series showed better docking scores than the Schiff base series. This suggests that cyclisation increases the docking scores. A closer look at the anti-tubercular results reveals that in Schiff base series (3a-I), increasing the substitution on nitrogen gave better activity (4a-I), perhaps due to increase in hydrophobicity resulting in better penetration of the Mtb cell wall. The structure - activity relationship of the compounds show that the presence of pharmacophoric moieties such as 1,3,4-thiadiazole nucleus (thiazolidinone moiety), increases antitubercular activity. A substituent, such as 4-methyl, 4-hydroxy group, attached to the phenyl ring and increases the substitution on nitrogen $\left(\mathrm{NH}_{2}\right.$ group) with thiazolidinone moiety to give higher activity, perhaps due to increase in hydrophobicity resulting in better penetration into the Mtb cell wall..

\section{CONCLUSION}

The overall outcome of these results reveals that thiazolidinone ring is a satisfactory backbone for antitubercular activity. These preliminary buy encouraging anti-tubercular results could offer an excellent framework in the field that may lead to the discovery of potent antitubercular agents.

\section{ACKNOWLEDGEMENT}

The authors are thankful to the VIT University, Vellore and Maratha Mandal's College of Pharmacy, Belgaum for providing facilities to carry out this work, and Maratha Mandal's Nathajirao G. Halgekar Institute of Dental Sciences and Research Centre, Belgaum for antitubercular activity.

\section{REFERENCES}

1. Loddenkemper R, Sagebiel D, Brendel A. Strategies against multidrug-resistant tuberculosis. Eur. Respir. J 2000; 20: 66-77.

2. Bastian IR, Colebunders $R$. Treatment and prevention of multidrug-resistant tuberculosis. Drugs 1999; 58: 633-661

3. Janin YL. Antituberculosis drugs: Ten years of research, Bioorg. Med. Chem 2007; 15: 24792513.

4. Rattan A, Kalia A, Ahmad N. Multidrug-resistant Mycobacterium tuberculosis: molecular perspectives. Emerg. Infect. Dis 1998; 4: 195203.

5. Karegoudar $P$, Prasad DJ, Ashok M, Mahalinga M, Poojary B, Holla BS. Synthesis, antimicrobial and anti-inflammatory activities of some 1,2,4triazolo[3,4-b][1,3,4]thiadiazoles and 1,2,4triazolo[3,4-b][1,3,4]thiadiazines bearing trichlorophenyl moiety. Eur. J. Med. Chem 2008; 43: 808-815.

6. Karakus $S$ and Rollas $S$. Synthesis and antituberculosis activity of new N-phenyl-N'-[4(5-alkyl/arylamino-1,3,4-thiadiazole-2yl)phenyl]thioureas. II Farmaco 2002; 57: 577581.

7. Foroumadi A, Kargar Z, Sakhteman A, Sharifzadeh $Z$, Feyzmohammadi $R$, Kazemi $M$, Shafiee $A$. Synthesis and antimycobacterial activity of some alkyl [5-(nitroaryl)-1,3,4-thiadiazol-2ylthio]propionates Bio. Org. Med. Chem. Letters 2006; 16: 1164-1167.

8. Capan G, Ulusoy N, Ergenc N, Kiraz M. New 6phenylimidazo[2,1-b]thiazole derivatives: Synthesis and antifungal activity. Monatsh. Chem 1999; 130: 1399-1407.

9. Vigorita MG, Ottana $R$, Monforte $F$, Maccari $R$, Trovato A, Monforte MT, Taviano MF. Synthesis and antiinflamatory, analgesic activity of 3,3'-(1,2-ethandiyl)-bis[2-aryl-4thiazolidinone] chiral compounds. Part 10. Bioorg. Med. Chem. Lett 2001; 11: 2791-2794.

10. Kavitha CV, Basappa S, Nanjunda S, Mantelingu K, Doreswamy S, Sridhar MA, Prasad JS, Rangappa KS. Synthesis os new bioactive venlafaxine analogs: Novel thiazolidin-4-ones as antimicrobials. Bioorg. Med. Chem 2006; 14: 2290-2299. 
11. Ottana R, Maccari R, Barreca ML, Bruno G, Rotondo A, Rossi A, Chiricosta G, Di Paola R, Sautebin L, Cuzzocrea S, Vigorita MG. 5-Arylidene-2imino-4-thiazolidinones: Design and synthesis of novel anti-inflammatory agents. Bioorg. Med. Chem 2005; 13: 4243-4252.

12. Kucukguzel G, Kocatepe A, De Clercq E, Sahin F, Gulluce M. Synthesis and biological activity of 4-thiazolidinones, thiosemicarbazides derived from diflunisal hydrazide. Eur. J. Med.Chem 2006; 41: 353-359.

13. Karigar AA, Himaja $M$, Mali SV, Jagadeesh $K P$, Sikarwar MS. One-Pot Synthesis and Antitubercular activity of 2-Amino-5-Aryl-5HThiazolo [4,3-b]-1,3,4-Thiadiazoles, International Research Journal of Pharmacy 2011; 2(1): 153-158.

14. Franzblau SG, Witzig RS, McLaughlin JC, Torres $P$, Madico G, Hernandez A, Degnan MT, Cook
MB, Quenzer VK, Ferguson RM, Gilman RH. Rapid, Low-Technology MIC Determination with Clinical Mycobacterium tuberculosis Isolates by Using the Microplate Alamar Blue Assay. J. Clin. Microbiol 1998; 36: 362-366.

15. Carvalho SA, Edson FD, Marcus VN, Maria CS, Felipe RV. Synthesis and antimycobacterial evaluation of new trans-cinnamic acid hydrazide derivatives. Bioorganic \& Medicinal Chemistry Letters 2008; 18: 538-541.

16. Reis RS, Neves JI, Lourenço SL, Fonseca LS, Lourenço MC. Comparison of Flow Cytometric and Alamar Blue Tests with the Proportional Method for Testing Susceptibility of Mycobacterium tuberculosis to Rifampin and Isoniazid. J. Clin. Microbiol 2004; 42: $2247-$ 2248. 